\title{
Marine n-3 Fatty Acids and Gene Expression in Peripheral Blood Mononuclear Cells
}

\author{
Stine M. Ulven • Mari C. Myhrstad • Kirsten B. Holven
}

Published online: 27 September 2014

(C) The Author(s) 2014. This article is published with open access at Springerlink.com

\begin{abstract}
Intake of marine n-3 fatty acids has been shown to have beneficial effects on cardiovascular disease. Gene expression analyses in peripheral blood mononuclear cells (PBMCs) are used to understand the underlying mechanisms of action of marine n-3 fatty acids. The aim of this review was to summarize the effects mediated by marine $n-3$ fatty acids on gene expression in PBMCs. A systematic literature search was conducted in PubMed in May 2014 and 14 papers were included. Targeted gene expression studies were reported in 9 papers and focused on genes involved in lipid metabolism and inflammation. Whole genome transcriptome analyses were conducted in 5 papers, and processes and pathways related to atherosclerotic plaque formation such as inflammation, oxidative stress response, cell cycle, cell adhesion, and apoptosis were modulated after fish oil supplementation. PBMC gene expression profiling has a potential to clarify further the molecular effects of fish oil consumption on human health.
\end{abstract}

Keywords Cardiovascular disease $\cdot$ Marine n-3 fatty acids . Dietary intervention study · Peripheral mononuclear cells . PBMCs · Inflammation · Lipid metabolism .

Transcriptomics $\cdot$ Gene expression

This article is part of the Topical Collection on Lipids

S. M. Ulven $(\bowtie) \cdot$ M. C. Myhrstad

Department of Health, Nutrition and Management, Faculty of Health Sciences, Oslo and Akershus University College of Applied Sciences (HiOA), P.O. Box 4, St Olavs plass, 0130 Oslo, Norway

e-mail: StineMarie.Ulven@hioa.no

K. B. Holven

Department of Nutrition, Institute for Basic Medical Sciences,

University of Oslo, P.O. Box 1046, Blindern, 0317 Oslo, Norway

\section{Introduction}

Fish consumption reduces the risk of developing cardiovascular disease (CVD) and CVD mortality [1,2]. Intervention trials with fish and fish oil containing the marine n-3 fatty acids eicosapentaenoic acid (EPA, 20:5 n-3) and docosahexaenoic (DHA, 22:6 n-3) have shown reduced total mortality and major coronary event including fatal and nonfatal MI [3-6]. However, recent meta-analyses and systematic reviews have shown that contradictory results exist regarding the beneficial effects of marine n-3 fatty acids on secondary prevention of coronary heart disease [7, 8]. The health beneficial effects of marine n-3 fatty acids are suggested to be mediated by reducing plasma triglycerides, reduced resting heart rate and blood pressure, and n-3 fatty acids may improve vascular function and immune response $[9,10]$. Most organizations and national health councils recommend regular intake of fatty fish (1-2 servings per week of fatty fish) in order to prevent CVD. Specific dietary recommendations for the marine n-3 fatty acids are not widely established and what those levels ought to be for particular populations are unclear.

Marine n-3 fatty acids regulate expression of genes involved in lipid metabolism and inflammation by acting as ligands for the peroxisomal proliferator-activated receptors (PPARs) [11]. In addition, fatty acids have the ability to reduce the activation of the transcription factor nuclear factor kappa $\mathrm{B}(\mathrm{NF}-\mathrm{kB})$ probably by interference with the PPARs $[12,13]$ or the Toll-like receptors $[14,15]$. Recently, the $\mathrm{G}$ protein-coupled receptor 120 (GPR120) has been characterized as n-3 fatty acid receptor/ sensor involved in mediating the anti-inflammatory effects of n-3 fatty acids [16]. The ability of marine n-3 fatty acids to alter gene expression may account for their potential health beneficial effects. To further understand the underlying molecular mechanisms of inclusion of fish oil and the marine $\mathrm{n}-3$ fatty acids in the human diet, the peripheral blood mononuclear cells (PBMCs) may, therefore, provide a model system for studying gene 
expression of mediators involved in the early development of atherosclerosis $[17,18]$. The PBMCs, which include monocytes and lymphocytes, are exposed to many of the same environmental factors as metabolic tissues and the arterial wall. PBMCs are also readily available, which make them suitable for studying gene expression in dietary intervention studies.

The aim of this review is to summarize the effects of marine $\mathrm{n}-3$ fatty acids on gene expression in PBMCs and discuss the findings in relation to human health.

Literature Search

A systematic literature search was conducted in Pubmed in May 2014 using the following terms: "(fish oil and gene expression and peripheral blood mononuclear cells), (fish oil and transcriptome and peripheral blood mononuclear cells), (fish oil and mRNA expression and peripheral blood mononuclear cells), (n-3 fatty acids and gene expression and peripheral blood mononuclear cells), (n-3 fatty acids and transcriptome and peripheral blood mononuclear cells), (n-3 fatty acids and mRNA expression and peripheral blood mononuclear cells), (fish oil and gene expression and mononuclear cells), (fish oil and transcriptome and mononuclear cells), (fish oil and mRNA expression and mononuclear cells), ( $\mathrm{n}-3$ fatty acids and gene expression and mononuclear cells), (n-3 fatty acids and transcriptome and mononuclear cells), (n-3 fatty acids and mRNA expression and mononuclear cells)". In total 157 instances of articles were identified, but after removing duplicates, 32 papers were screened by reading abstracts (Fig. 1). In total 16 papers were excluded based on the following criteria of not be a human study, not using fish oil or marine n-3 fatty acids, no gene expression data, not an original paper (Fig. 1). After reading 16 full-text papers, 2 more papers were excluded because there was no effect of fish oil or $n-3$ fatty acids on PBMC gene expression was shown in these papers (Fig. 1). In total, 14 original papers were included in the review.

\section{Fish Oil Intervention Studies and Targeted Gene Expression Studies in PBMCs}

Table 1 summarizes the effect of marine n-3 fatty acids from 9 targeted gene expression studies in PBMCs. In total, 7 fish oil intervention studies have used PBMC gene expression data of which one was a postprandial study (Table 1). The duration of the studies ranged from 4-12 weeks. Kaminski et al studied for the first time in 1993 the effect of marine n-3 fatty acids on plateletderived growth factor (PDGF) gene expression in human mononuclear cells (MNC) [19]. In a controlled randomized, observerblinded study with 14 healthy males, intake of $7 \mathrm{~g} / \mathrm{d}$ of fish oil concentrate (54\% EPA and $28.7 \%$ DHA) reduced the mRNA level of PDGF-A and B after both 1 and 6 weeks of intervention

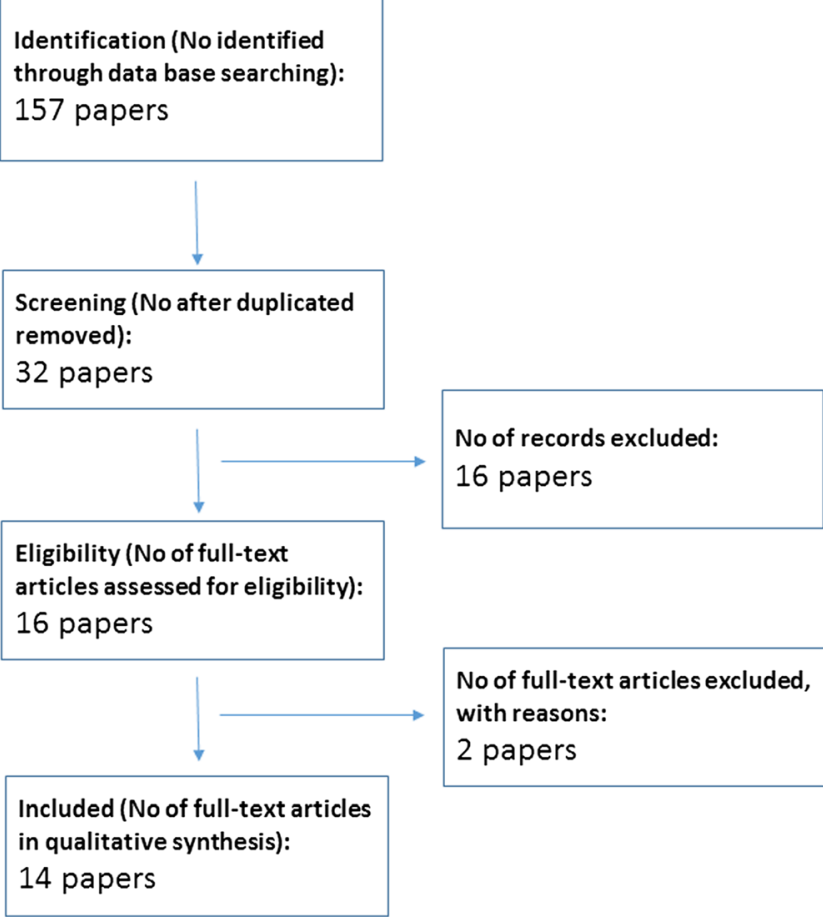

Fig. 1 A flow chart of the PubMed search and selection of papers

(6 out of 7 subjects) [19]. In the control, group, receiving no supplement, the expression level of PDGF-A remained unaltered in 6 out of 7 subjects. To further examine other growth factors, known to be involved in proliferation of endothelial cells, Jendraschak et al analyzed the mRNA expression level of platelet-derived endothelial cell growth factor (PD-ECGF), insulin-like growth factor (IGF-1A, 1B), and transforming growth factor- $\beta 1$ (TGF- $\beta 1$ ) [20]. The expression level of PDECGF was constant, whereas gene expression of TGF- $\beta 1$, IGF$1 \mathrm{~A}$, and IGF-1B were varying over time and among subjects, independently of each other [20]. Baumann et al performed a 4week randomized, investigator-blinded intervention trial with $7 \mathrm{~g} / \mathrm{d}$ of fish oil concentrate (41.4 \% EPA and $23.6 \%$ DHA) $(n=7), 7 \mathrm{~g} / \mathrm{d}$ of corn oil concentrate $(50.1 \%$ linoleic acid) $(n=7)$, or $7 \mathrm{~g} / \mathrm{d}$ of olive oil concentrate ( $60.5 \%$ oleic acid) $(n=7)$ vs no dietary intervention $(n=7)$ [21]. The MNC gene expression levels of PDGF-A, PDGF-B, and monocyte chemoattractant protein-1 (MCP-1), also called chemokine (C-C motif) ligand 2 (CCL2), were reduced in the fish oil group in accordance with the data from Kaminski et al [19]. Interleukin-10 (IL-10) and heparin-bound epidermal growth factor (HB-EGF) mRNA levels were not changed by fish oil supplementation [21]. The MNC mRNA levels of PDGF-A, PDGF-B, MCP-1, IL-10, and HBEGF were not altered in the n-6 and n-9 fatty acid groups [21]. PDGF is a chemoattractant for neutrophils, monocytes, fibroblasts, and smooth muscle cells. Proliferation of vascular endothelial and intimal smooth muscle cells, characteristic for atherosclerotic lesions, may be initiated and maintained by PDGF-like protein secreted by monocytes, and fish oil consumption seems 


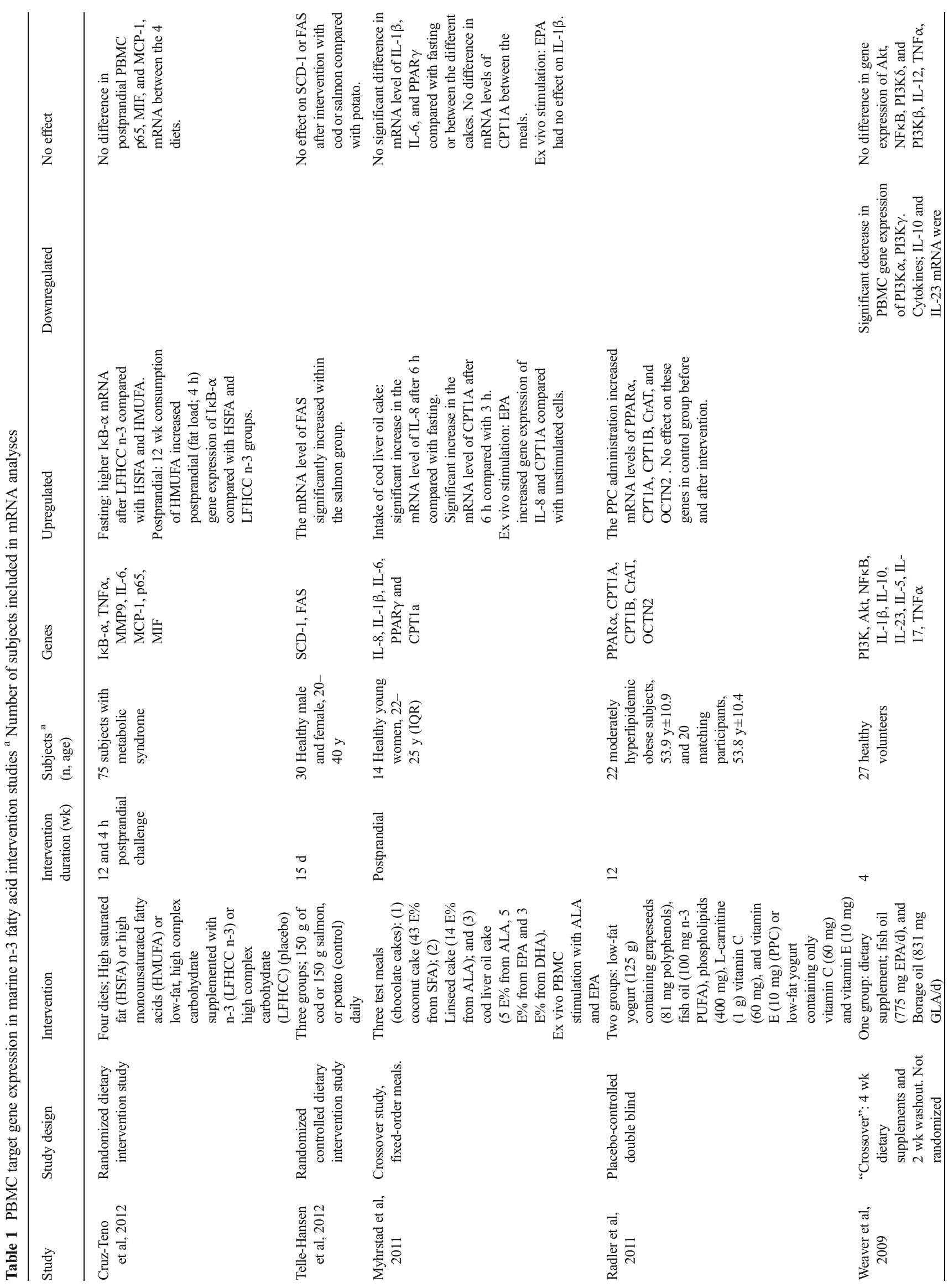




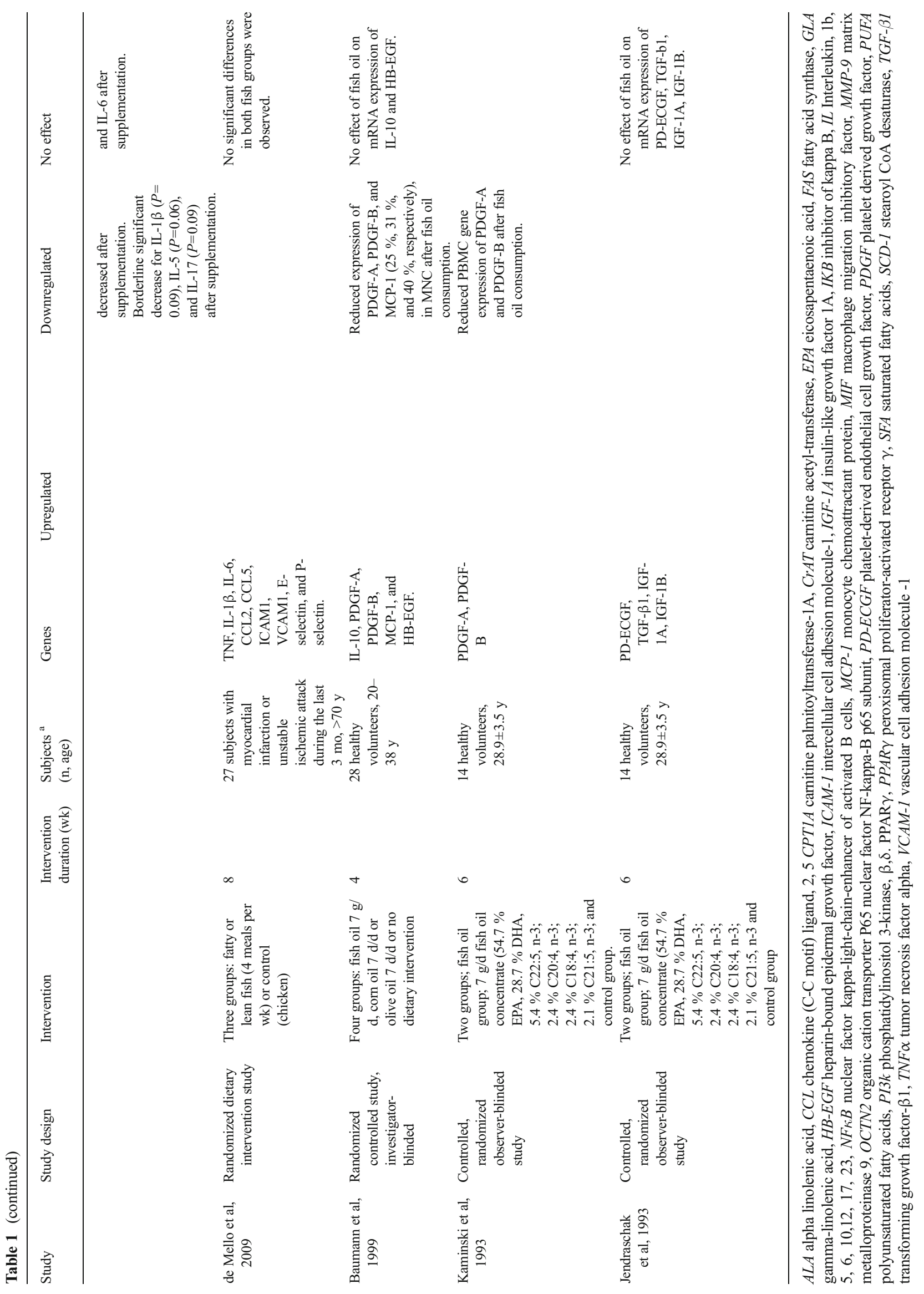


to regulate the expression of this gene in PBMCs, which may have a health beneficial effect on development of atherosclerosis.

Weaver et al performed a dietary "cross-over" study in which 27 healthy adults were given a controlled background diet together with fish oil (775 mg EPA/d) and borage oil (831 mg $\gamma$-linolenic acid (GLA18:3, n-6)/d) for 4 weeks followed by a 2- week wash-out period [22]. Supplementation of fish oil and borage oil induced a decrease in the expression of phosphatidylinositol 3-kinase (PI3K) $\alpha$ and PIK3K $\gamma$ [22]. The expression of PI3K $\beta, \mathrm{PI} 3 \mathrm{~K} \delta$, protein kinase $\mathrm{B}$, also known as Akt and NF-kB was not significantly altered during supplementation [22]. PI3K $\alpha$ and PIK3K $\gamma$ play an important roles in eicosanoid formation and in cell growth, survival, and inflammation by modulating Akt and NF- $\mathrm{kB}$ signaling, which in turn influence the production of a variety of signaling molecules such as cytokines. Furthermore, 8 cytokines known to play an important role in the inflammatory response were measured in the same study. The mRNA expression level of interleukin(IL)-1 $\beta$, IL-10, and IL-23 were significantly decreased after supplementation with fish oil and borage oil [22]. In addition, the expression of IL-5 and IL-17 showed trends toward decreased expression after supplementation, whereas the expression of TNF- $\alpha$ and IL- 6 were not changed [22]. This study shows that 4-week supplementation with fish oil and borage oil reduces the expression of PI $3 \mathrm{~K} \alpha$ and PIK $3 \mathrm{~K} \gamma$, which are early steps in cellular signal transduction, as well as reduce expression of several important downstream genes such as different interleukins. If the effect is mediated via altered formation of inflammatory eicosanoids or a direct effect on gene regulation is not known. It cannot be ruled out, that the effects may also have been caused by a change in $n-6$ and $n-3$ PUFA content and not only mediated by the marine $n-3$ fatty acids.

In a placebo-controlled double blind study with 22 moderately hyperlipidemic obese humans consuming low-fat yogurt enriched with a combination of low-dose PUFAs from fish oil (100 mg n-3 fatty acids), polyphenols and L-carnitine (PPC) twice a day for 12 weeks, the expression of genes involved in lipid metabolism were compared with 20 matching participants ingesting low-fat yogurt [23]. The level of plasma free fatty acids and triglycerides were significantly reduced in the PPC group. In addition, the PBMC gene expression of PPAR $\alpha$, carnitine palmitoyltransferase-1, CPT1A, and CPT1B, carnitine acetyl-transferase (CrAT) and organic cation transporter 2 (OCTN2) was significantly increased in the PPC group [23]. No change was seen in the control group [23]. This study showed that a reduction in plasma free fatty acids and triglycerides coincided with increased PBMC mRNA expression level of genes encoding proteins involved in fatty acid oxidation among subjects consuming PCC. Because CPT1 is regulating the capacity for mitochondrial fatty acid oxidation, the increase in mRNA expression of CPT1 may stimulate oxidation and result in the subsequent observed decrease in free fatty acids. The observed increase in the
mRNA level of PPAR $\alpha$ may also partly explain the increased expression of CPT1, CPT2, and OCTN2 [24]. However, the effects seen in this study may also be related to intake of carnitine and not only mediated by the marine $\mathrm{n}-3$ fatty acids.

Cruz-Teno et al performed a randomized dietary intervention study with 75 subjects with metabolic syndrome allocated to 1 of 4 diets; high saturated fatty acids (HSFA); high monounsaturated fatty acids (HMUFA) and 2 low-fat, high complex carbohydrate (LFHCC) diets, supplemented with n-3 fatty acids (LFHCC n-3) or placebo (LFHCC), for 12 weeks, followed by a postprandial challenge [25•]. In the HMUFA diet group and 4 hours after the fat overload, a significant postprandial increase in the mRNA expression level of nuclear factor of kappa light polypeptide gene enhancer in B-cells inhibitor, alpha (IkB)- $\alpha$ compared with HSFA and LFHCC n3 diets was observed [25•]. In contrast, at fasting levels, after 12 weeks consumption of LFHCC n-3 diet, the expression level of IKB- $\alpha$ mRNA was higher compared with HSFA and HMUFA diets. Intake of the 4 different fat load breakfasts induced an increase in the mRNA expression of TNF $\alpha$, metalloproteinase 9 (MMP-9), and IL-6 independently of the diet consumed, reflecting an acute inflammatory response during the postprandial period. No changes in NF-KB p65 subunit (p65), macrophage migration inhibitory factor (MIF) and MCP-1 mRNA levels were observed after intake of any of the 4 different diets [25•]. The postprandial plasma protein concentration of MCP-1 was reduced after intake of HMUFA and LFHCC n-3 diets compared with HSFA diet. After the fat load, an increase in the postprandial plasma protein levels of IL-6 independently of diet consumed was observed. No effects were found by diet on plasma protein level of TNF $\alpha$ $[25 \bullet]$.

We have also performed a postprandial study where 3 different test meals were consumed by 14 healthy women in a fixed order with 14 days wash-out between each test meal [26•]. The test meals consisted of 3 different $150 \mathrm{~g}$ chocolate cakes enriched with coconut fat [43\% energy as saturated fat and $1 \%$ energy as alpha-linolenic acid (ALA)], linseed oil (14\% energy as ALA and $30 \%$ energy as saturated fat), and cod liver oil (5\% energy as EPA and DHA and $5 \%$ energy as ALA in addition to $31 \%$ energy as saturated fat). At 3 hours after intake of the test meal, the plasma triglycerides increased in all groups, whereas no significant differences in triglycerides were observed after 6 hours compared with baseline in any of the groups. The mRNA expression level of IL- 8 was significantly upregulated after intake of the cod liver oil cake at 6 hours compared with fasting level. This increase was significantly different from the effect observed after intake of linseed oil cake. No increase in plasma protein level of IL-8 was observed for any of the test meals. The mRNA level of CPT1A was also significantly increased 6 hours after intake of cod liver oil cake compared with 3 hours. In addition, ex vivo cultured PBMCs were incubated for 24 hours with 
$60 \mu \mathrm{M}$ of EPA. Incubation with EPA significantly increased the mRNA expression level of IL-8 and CPT1A compared with unstimulated cells [26•].

\section{Fish Intervention Studies and Targeted Gene Expression Studies in PBMCs}

Of the total 9 papers on targeted gene expression analysis, 2 dietary intervention studies with fatty or lean fish have been reported. De Mello et al showed in a 8-week randomized study with 3 groups, fatty fish diet $(n=11,0.89 \mathrm{~g}$ EPA+ $\mathrm{DHA} / \mathrm{d})$, lean fish diet $(n=11,0.43 \mathrm{~g} \mathrm{EPA}+\mathrm{DHA} / \mathrm{d})$ and control diet ( $n=6,0.18 \mathrm{~g}$ EPA+DHA/d) among patients with coronary heart disease, that 4 portions of fish per week for 8 weeks did not differently change the gene expression of TNF, IL-1 $\beta$, IL-6, chemokine (C-C motif) ligand 2 (CCL-2), CCL-5, intercellular cell adhesion molecule-1 (ICAM1), vascular cell adhesion molecule -1 (VCAM1), and E- and Pselectin during the intervention in neither the fatty fish or the lean fish group [27]. We have also shown in a 15-day randomized study with 3 groups, fatty fish diet $(n=11,3.1 \mathrm{~g}$ $\mathrm{EPA}+\mathrm{DHA} / \mathrm{d})$, lean fish diet $(n=9,0.13 \mathrm{~g} \mathrm{EPA}+\mathrm{DHA} / \mathrm{d})$, and control diet $(n=10,0 \mathrm{~g}$ EPA+DHA/d) among healthy adults, that $150 \mathrm{~g}$ of fish per day did not change the gene expression of stearoyl-CoA desaturase-1 (SCD-1) and fatty acid synthase (FAS) in PBMCs compared with the control group [28]. We did, however, observe an increase in FAS mRNA level within the salmon group. The plasma triglyceride levels were significantly decreased in both fish groups compared with the control group, and HDL-cholesterol was significantly increased after intake of salmon compared with the control group. Total-cholesterol and LDL-cholesterol were not changed between or within any of the groups during the intervention [28].

Overall, few targeted gene expression studies have been performed and a limited number of genes related to lipid metabolism (such as PPAR $\alpha$, CPT1, FAS, and SCD-1) have been examined. Most of the effects seen are related to genes involved in immune function. In many of these studies the effect has been studied within the group receiving fish oil and not in comparison with the control group.

Fish Oil Interventions and Whole Genome Transcriptomics in PBMCs

PBMC transcriptome analyses in human intervention studies with fish oil were reported in 5 papers and are summarized in Table 2. Three of the studies were double-blinded randomized placebo-controlled studies and all used fish oil $(1.6 \mathrm{~g} / \mathrm{d}$ EPA+ DHA/d-3 g/d EPA+DHA) and included healthy adults or elderly subjects, subjects with Alzheimer disease and obese insulin resistant subjects. The duration of the studies ranged from 6-26 weeks (Table 2).

Bouwens et al [29] first reported the effect of fish oil supplementation on whole genome gene expression profiles in human PBMCs in 2009. A double-blinded randomized intervention trial in an elderly population was performed. The triglyceride levels were significantly reduced in the fish oil group compared to the high oleic sunflower oil (HOSO, control) group, but no change in CRP level was observed in any of the groups. Within the fish oil group consuming $1.8 \mathrm{~g} / \mathrm{d} \mathrm{EPA}+$ DHA $(n=23), 1040$ gene transcripts were regulated after 26 weeks of intervention. Within the high oleic sunflower oil (HOSO, control) group $(n=25), 298$ genes were regulated after 26 weeks of intervention. In total 140 genes were overlapping between the groups, and the direction of change was the same in both groups. Pathways analysis (using GenMAPP) showed that fish oil supplementation for 26 weeks significantly decreased the expression of genes involved in inflammatory pathways, such as eicosanoid synthesis, interleukin signaling, and MAP kinase signaling. Fish oil supplementation also significantly decreased expression of genes related to atherosclerotic processes, such as cell adhesion, scavenger receptor activity, and adipogenesis. Other pathway analysis and gene set enrichment analyses (GSEA analysis) showed a decrease in similar inflammatory signaling pathways as described above in the fish oil group. In addition, these analyses also showed that genes involved in nuclear transcription factor $\mathrm{kB}(\mathrm{NF}-\mathrm{kB})$ and Toll-like receptor signaling were decreased in the fish oil group. A decrease in oxidative stress, cell adhesion, cell cycle, PPAR and LXR/RXR activation, and hypoxia signaling was also observed in the fish oil group. In the HOSO group, pathways analysis showed a decreased expression of genes involved in inflammation and cell adhesion [29].

Vedin et al showed in a 24-week double-blinded randomized, placebo-controlled fish oil study (2.3 g/d EPA+DHA) among 16 subjects with Alzheimer disease that 19 genes (with a false discovery rate of $10 \%$ ) were changed in the fish oil group [30•]. In total, 9 genes were upregulated by the fish oil supplementation, and of these, 1 gene was also upregulated in the control group (corn oil), and 1 gene was significantly different in fold change between the 2 groups (CD63 molecule). In total, 10 genes were downregulated by the fish oil supplementation, and of these, 4 genes were significantly different in fold change between the groups [Ras homolog gene family, member B (RHOB), LOC 3999491 protein (LOC399491), zinc finger protein 24 (ZNF24), and Anaphase promoting complex subunit 5 (ANAPC5)]. Many of the modulated genes are involved in inflammation regulation, and neurodegeneration and in ubiquitination processes [30•].

Rudkowska et al showed in a 8 weeks randomized controlled crossover study with fish oil (1.8 g/d EPA+DHA) and fish oil plus fish gelatin (+FG) with 16 obese insulin-resistant subjects that 805 genes were regulated in the fish oil group 


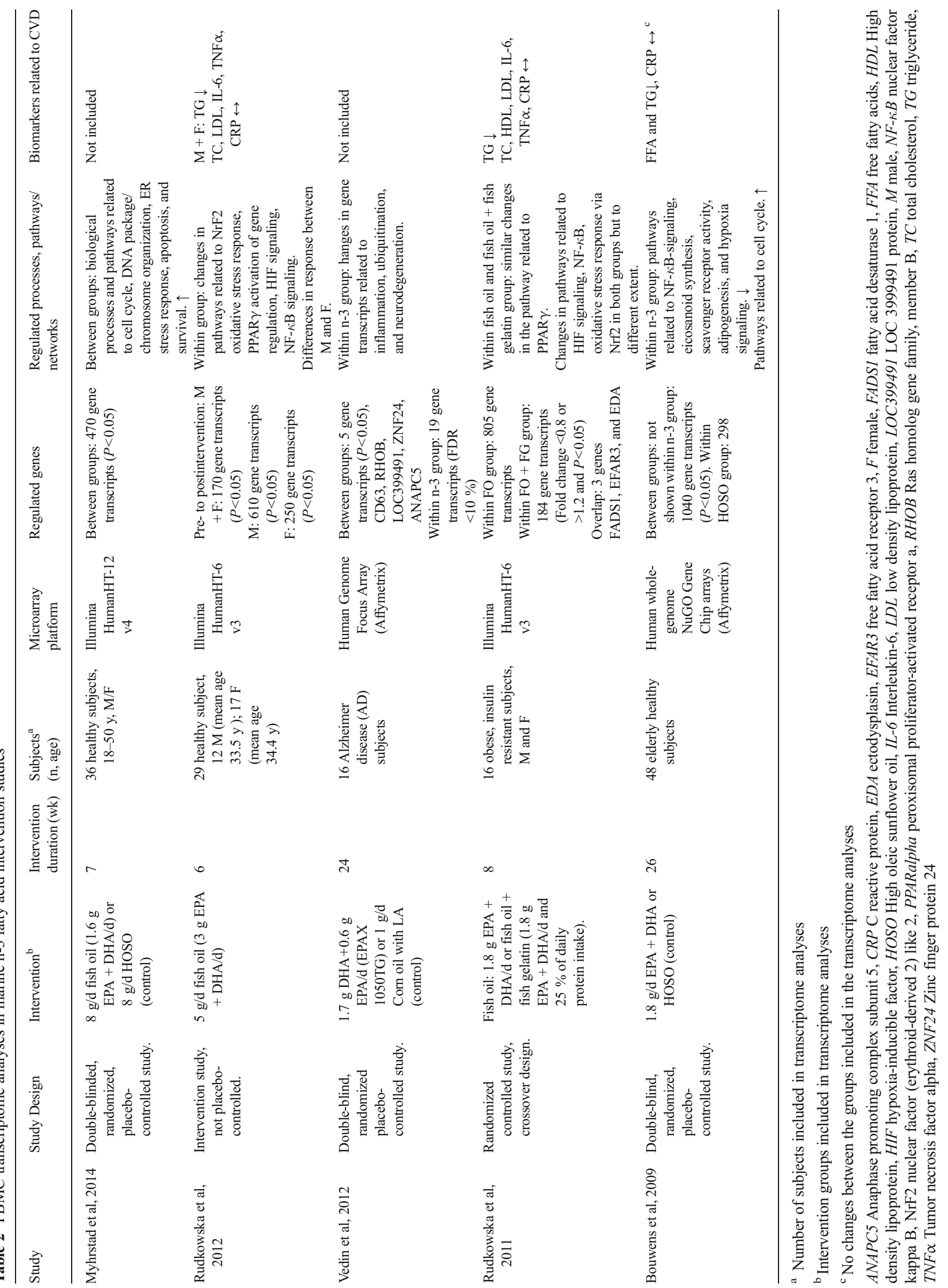


[31]. A decrease in fasting triglyceridesin both groups, but no changes in total cholesterol, LDL-cholesterol, HDL-cholester$\mathrm{ol}$, and circulating inflammatory markers such as CRP, IL-6, and TNF $\alpha$ were observed. Among the 805 genes, $65 \%$ (524) were downregulated and $35 \%$ (281) were upregulated. In the fish oil plus fish gelatin group, 184 genes were regulated after an 8-week intervention (59\% (109) downregulated and $41 \%$ (75) were upregulated). Of these genes, only 3 genes were overlapping in both supplementation periods [fatty acid desaturase 1 (FADS1), free fatty acid receptor 3 (EFAR3), and ectodysplasin (EDA)]. Pathways analysis (Ingenuity) showed that oxidative stress response mediated by nuclear factor (erythroid-derived 2) like $2(\mathrm{NrF} 2)$, PPAR $\alpha$, hypoxiainducible factor (HIF) and NF-KB signaling pathways were changed in both groups during the intervention but to different extents [31]. Rudkowska et al have also investigated the PBMC gene expression changes following fish oil supplementation ( $3 \mathrm{~g} / \mathrm{d}$ EPA+DHA) for 6 weeks among 29 healthy adults (12 male and 17 females) [32]. The study was not a randomized study and was without a control group. They found that 170 genes were regulated after a 6 -week intervention (47\% (80) downregulated and $53 \%$ (90) upregulated). In male subjects, 610 genes were differently expressed (46\% (283) down-regulated and $54 \%$ (327) upregulated) by fish oil supplementation. In females, only 250 genes were differently expressed (49\% (122) downregulated and $51 \%$ (128) upregulated) by fish oil supplementation. Of these genes, only 9 genes were overlapping. Pathways analysis (Ingenuity) showed that oxidative stress response genes mediated by $\mathrm{NrF} 2, \operatorname{PPAR} \alpha, \mathrm{HIF}$ and NF-kB signaling pathway were changed among males and females but to different extend. A decrease in fasting plasma triglycerides among males and females was observed, however, no other changes in total cholesterol, LDL-cholesterol, and circulating inflammatory markers such as CRP, IL-6, TNF $\alpha$ was observed [32].

In a 7-week double-blinded randomized controlled fish oil study (1.6 g/d EPA+DHA) with healthy adults, we have recently observed that 470 genes $(P<0.05)$ were differently expressed between the fish oil group and the HOSO group [33••]. Two hundred-and thirty-six genes were upregulated and 234 were downregulated in the fish oil group compared with the HOSO group. Among the upregulated genes, several biological processes were enriched such as cell cycle and DNA packing and chromosome organization. We found that 11 genes were significantly changed in the fish oil group (FDR q-value $<10 \%$ ), and none in the HOSO group. Gene set enrichment analysis (GSEA) showed that in particular cell cycle, apoptosis, immune response, protein folding and maturation and DNA damage were significantly regulated in the fish oil group compared with the HOSO group. Moreover, Gene transcripts with common motifs for 35 known transcription factors including E2F, TP53, HIF, and ATF4 were upregulated after intake of fish oil compared with HOSO [33••].
Overall, 5 papers have reported using whole genome transcriptomics in fish oil intervention studies. Annotation and pathways analysis showed that fish oil supplementation modulated the expression of genes involved in inflammatory pathways, such as eicosanoid synthesis, interleukin signaling, and MAP kinase signaling, oxidative stress response, cell cycle, cell adhesion, apoptosis, scavenger receptor activity, adipogenesis, protein folding, maturation, and DNA damage [29-32, 33••]. Many of these are anti-atherogenic and anti-inflammatory genes, which play a role in several processes involved in atherosclerotic plaque formation. PBMCs, consisting of monocytes and lymphocytes are cells central in these processes including adhesion, infiltration, and foam cell formation, and, therefore, it seems like whole genome transcriptomics can be used to identify novel pathways modulated by intake of fish oil. Data from fish oil supplementation studies further indicates that these effects may be mediated via many transcription factors and signaling pathways, such as NF-KB, Toll-like receptor signaling, PPAR and LXR/RXR activation and hypoxia signaling [29, 33••]. The studies summarized in this paper have different duration, doses and study subjects, but overall they show that fish oil supplementation may influence several biological pathways and processes via different transcription factors. The discrepancy between the studies by Bouwens et al [29] and Rudkowska et al [31, 32] on PPAR $\alpha$ activation, may be caused by differences in the duration of the studies or the age and genotype of the study subjects. Because it has been shown that the response of fish oil on triglycerides depends on sex and genotype [34], genetic differences may cause the variation in gene expression responses observed in these intervention studies. Another explanation could be that changes in gene expression are reflecting the response of PBMCs to EPA-DHA-induced systemic adaptations in the body and may, therefore, not be a direct effect of EPA+DHA on mononuclear cells. The major limitation in these transcriptomic studies is that most of the studies (4 out of 5) analyzed the within group changes, rather than between group differences.

\section{Conclusions}

PBMC gene expression analysis in human dietary intervention studies with fish oil or fatty fish can be a powerful tool to understand the underlying molecular mechanisms explaining the association between high intakes of marine n-3 fatty acids and effects on morbidity and mortality from CVD. These studies show the potential of PBMC gene expression profiling to investigate the effects of nutrition on human health. Gene expression profiling seems to be more sensitive to fish oil interventions than the traditional biochemical parameters measured in circulation and enable us to study gene expression of mediators involved in the early development of atherosclerosis. Future studies should integrate gene expression patterns 
with biochemical parameters to determine the precise mechanisms of action of EPA and DHA in humans. More emphasis should also be on examining the gene expression profiles in PBMCs in responders versus non-responders in order to understand the influence of genetic factors on the effect of fish oil on lipids and inflammatory markers.

Acknowledgments This work was supported by Oslo and Akershus University College of Applied Sciences, Department of Health, Nutrition and Management and the University of Oslo, Department of Nutrition.

\section{Compliance with Ethics Guidelines}

Conflict of Interest Stine Ulven, Kirsten Holven, and Mari Myhrstad have no conflicts of interest.

Human and Animal Rights and Informed Consent This article does not contain any studies with human or animal subjects performed by the author.

Open Access This article is distributed under the terms of the Creative Commons Attribution License which permits any use, distribution, and reproduction in any medium, provided the original author(s) and the source are credited.

\section{References}

Papers of particular interest, published recently, have been highlighted as:

- Of importance

•. Of major importance

1. Hu FB, Bronner L, Willett WC, Stampfer MJ, Rexrode KM, Albert $\mathrm{CM}$, et al. Fish and omega-3 fatty acid intake and risk of coronary heart disease in women. JAMA. 2002;287:1815-21.

2. He K, Song Y, Daviglus ML, Liu K, Van Horn L, Dyer AR, et al. Accumulated evidence on fish consumption and coronary heart disease mortality: a meta-analysis of cohort studies. Circulation. 2004;109:2705-11.

3. Burr ML, Fehily AM, Gilbert JF, Rogers S, Holliday RM, Sweetnam PM, et al. Effects of changes in fat, fish, and fibre intakes on death and myocardial reinfarction: diet and reinfarction trial (DART). Lancet. 1989;2:757-61.

4. Dietary supplementation with $n-3$ polyunsaturated fatty acids and vitamin E after myocardial infarction: results of the GISSIPrevenzione trial. Gruppo Italiano per lo Studio della Sopravvivenza nell'Infarto miocardico. Lancet. 1999;354:447-55.

5. Tavazzi L, Maggioni AP, Marchioli R, Barlera S, Franzosi MG, Latini R, et al. Effect of $n-3$ polyunsaturated fatty acids in patients with chronic heart failure (the GISSI-HF trial): a randomised, double-blind, placebo-controlled trial. Lancet. 2008;372:1223-30.

6. Yokoyama M, Origasa H, Matsuzaki M, Matsuzawa Y, Saito Y, Ishikawa Y, et al. Effects of eicosapentaenoic acid on major coronary events in hypercholesterolaemic patients (JELIS): a randomised open-label, blinded endpoint analysis. Lancet. 2007;369:1090-8.

7. Rizos EC, Ntzani EE, Bika E, Kostapanos MS, Elisaf MS. Association between omega-3 fatty acid supplementation and risk of major cardiovascular disease events: a systematic review and meta-analysis. JAMA. 2012;308:1024-33.
8. Wen YT, Dai JH, Gao Q. Effects of Omega-3 fatty acid on major cardiovascular events and mortality in patients with coronary heart disease: a meta-analysis of randomized controlled trials. Nutr Metab Cardiovasc Dis. 2014;24:470-5.

9. Mozaffarian D, Wu JH. Omega-3 fatty acids and cardiovascular disease: effects on risk factors, molecular pathways, and clinical events. J Am Coll Cardiol. 2011;58:2047-67.

10. Myhrstad MC, Retterstøl K, Telle-Hansen VH, Ottestad I, Halvorsen B, Holven KB, et al. Effect of marine n-3 fatty acids on circulating inflammatory markers in healthy subjects and subjects with cardiovascular risk factors. Inflamm Res. 2011;60:30919.

11. Lee CH, Olson P, Evans RM. Mini-review: lipid metabolism, metabolic diseases, and peroxisome proliferator-activated receptors. Endocrinology. 2003;144:2201-7.

12. Castrillo A, Joseph SB, Marathe C, Mangelsdorf DJ, Tontonoz P. Liver $\mathrm{X}$ receptor-dependent repression of matrix metalloproteinase9 expression in macrophages. J Biol Chem. 2003;278:10443-9.

13. Joseph SB, Castrillo A, Laffitte BA, Mangelsdorf DJ, Tontonoz P. Reciprocal regulation of inflammation and lipid metabolism by liver X receptors. Nat Med. 2003;9:213-9.

14. Lee JY, Plakidas A, Lee WH, Heikkinen A, Chanmugam P, Bray G, et al. Differential modulation of Toll-like receptors by fatty acids: preferential inhibition by $\mathrm{n}-3$ polyunsaturated fatty acids. J Lipid Res. 2003;44:479-86.

15. Wong SW, Kwon MJ, Choi AM, Kim HP, Nakahira K, Hwang DH. Fatty acids modulate Toll-like receptor 4 activation through regulation of receptor dimerization and recruitment into lipid rafts in a reactive oxygen species-dependent manner. J Biol Chem. 2009;284:27384-92.

16. Oh DY, Talukdar S, Bae EJ, Imamura T, Morinaga H, Fan W, et al. GPR120 is an omega-3 fatty acid receptor mediating potent antiinflammatory and insulin-sensitizing effects. Cell. 2010;142:68798.

17. Pasterkamp G, Daemen M. Circulating cells: the biofactory for markers of atherosclerotic disease. Eur Heart J. 2008;29:2701-2.

18. Visvikis-Siest S, Marteau JB, Samara A, Berrahmoune H, Marie B, Pfister M. Peripheral blood mononuclear cells (PBMCs): a possible model for studying cardiovascular biology systems. Clin Chem Lab Med. 2007;45:1154-68.

19. Kaminski WE, Jendraschak E, Kiefl R, von Schacky C. Dietary omega-3 fatty acids lower levels of platelet-derived growth factor mRNA in human mononuclear cells. Blood. 1993;81:1871-9.

20. Jendraschak E, Kaminski WE, Hessel F, Kiefl R, von Schacky C. Growth factor mRNA profiles in unstimulated human mononuclear cells: identification of genes which are constitutively and variably expressed. Biochem Biophys Res Commun. 1993;196:25-31.

21. Baumann KH, Hessel F, Larass I, Müller T, Angerer P, Kiefl R, et al. Dietary omega-3, omega- 6 , and omega- 9 unsaturated fatty acids and growth factor and cytokine gene expression in unstimulated and stimulated monocytes. A randomized volunteer study. Arterioscler Thromb Vasc Biol. 1999;19:59-66.

22. Weaver KL, Ivester P, Seeds M, Case LD, Arm JP, Chilton FH Effect of dietary fatty acids on inflammatory gene expression in healthy humans. J Biol Chem. 2009;284:15400-7.

23. Radler U, Stangl H, Lechner S, Lienbacher G, Krepp R, Zeller E, et al. A combination of $(\omega-3)$ polyunsaturated fatty acids, polyphenols and L-carnitine reduces the plasma lipid levels and increases the expression of genes involved in fatty acid oxidation in human peripheral blood mononuclear cells and HepG2 cells. Ann Nutr Metab. 2011;58:133-40.

24. Ringseis R, Luci S, Spielmann J, Kluge H, Fischer M, Geissler S, et al. Clofibrate treatment up-regulates novel organic cation transporter (OCTN)-2 in tissues of pigs as a model of non-proliferating species. Eur J Pharmacol. 2008;583:11-7. 
25. Cruz-Teno C, Pérez-Martínez P, Delgado-Lista J, Yubero-Serrano EM, García-Ríos A, Marín C, et al. Dietary fat modifies the postprandial inflammatory state in subjects with metabolic syndrome: the LIPGENE study. Mol Nutr Food Res. 2012;56:854-65. This study showed that the expression level of I $K B-\alpha$ was increased after intake of a low-fat, high complex carbohydrate diet supplemented with $n-3$ fatty acids. A fat load also increased the PBMC expression of inflammatory markers.

26. Myhrstad MC, Narverud I, Telle-Hansen VH, Karhu T, Lund $\mathrm{DB}$, Herzig $\mathrm{KH}$, et al. Effect of the fat composition of a single high-fat meal on inflammatory markers in healthy young women. Br J Nutr. 2011;106:1826-35. This study showed that intake of fish oil modulates PBMC gene expression in a postprandial study.

27. de Mello VD, Erkkilä AT, Schwab US, Pulkkinen L, Kolehmainen M, Atalay M, et al. The effect of fatty or lean fish intake on inflammatory gene expression in peripheral blood mononuclear cells of patients with coronary heart disease. Eur J Nutr. 2009;48:44755 .

28. Telle-Hansen VH, Larsen LN, Høstmark AT, Molin M, Dahl L, Almendingen K, Ulven SM. Daily intake of cod or salmon for 2 weeks decreases the 18:1n-9/18:0 ratio and serum triacylglycerols in healthy subjects. Lipids. 2012;47:151-60.

29. Bouwens M, van de Rest O, Dellschaft N, Bromhaar MG, de Groot LC, Geleijnse JM, et al. Fish-oil supplementation induces antiinflammatory gene expression profiles in human blood mononuclear cells. Am J Clin Nutr. 2009;90:415-24.
30. Vedin I, Cederholm T, Freund-Levi Y, Basun H, Garlind A, Irving GF, et al. Effects of DHA-rich n-3 fatty acid supplementation on gene expression in blood mononuclear leukocytes: the OmegaD study. PLoS One. 2012;7:e35425. This paper showed that genes involved in inflammatio, regulation of neurodegeneration and in ubiquitination processes were regulated by fish oil among subjects with Alzheimer disease.

31. Rudkowska I, Ponton A, Jacques H, Lavigne C, Holub BJ, Marette A, et al. Effects of a supplementation of $n-3$ polyunsaturated fatty acids with or without fish gelatin on gene expression in peripheral blood mononuclear cells in obese, insulin-resistant subjects. J Nutrigenet Nutrigenomics. 2011;4:192-202.

32. Rudkowska I, Paradis AM, Thifault E, Julien P, Tchernof A, Couture P, et al. Transcriptomic and metabolomic signatures of an n-3 polyunsaturated fatty acids supplementation in a normolipidemic/normocholesterolemic Caucasian population. J Nutr Biochem. 2013;24:54-61.

33.• Myhrstad MC, Ulven SM, Günther CC, Ottestad I, Holden M, Ryeng E, et al. Fish oil supplementation induces expression of genes related to cell cycle, endoplasmic reticulum stress and apoptosis in peripheral blood mononuclear cells: a transcriptomic approach. J Intern Med. 2014. doi:10.1111/joim.12217. This study compared whole genome gene expression analysis between a fish oil and a control group.

34. Caslake MJ, Miles EA, Kofler BM, Lietz G, Curtis P, Armah CK, et al. Effect of sex and genotype on cardiovascular biomarker response to fish oils: the FINGEN study. Am J Clin Nutr. 2008;88(3):618-29. 